\title{
Arnowitt-Deser-Misner representation and Hamiltonian analysis of covariant renormalizable gravity
}

\author{
Masud Chaichian, 1 Markku Oksanen, 2 Anca Tureanu 3 \\ Department of Physics, University of Helsinki, P.O. Box 64, \\ FI-00014 Helsinki, Finland
}

\begin{abstract}
We study the recently proposed Covariant Renormalizable Gravity (CRG), which aims to provide a generally covariant ultraviolet completion of general relativity. We obtain a space-time decomposed form - an Arnowitt-Deser-Misner (ADM) representation - of the CRG action. The action is found to contain time derivatives of the gravitational fields up to fourth order. Some ways to reduce the order of these time derivatives are considered. The resulting action is analyzed using the Hamiltonian formalism, which was originally adapted for constrained theories by Dirac. It is shown that the theory has a consistent set of constraints. It is, however, found that the theory exhibits four propagating physical degrees of freedom. This is one degree of freedom more than in Hořava-Lifshitz (HL) gravity and two more propagating modes than in general relativity. One extra physical degree of freedom has its origin in the higher order nature of the CRG action. The other extra propagating mode is a consequence of a projectability condition similarly as in HL gravity. Some additional gauge symmetry may need to be introduced in order to get rid of the extra gravitational degrees of freedom.
\end{abstract}

PACS: 04.50.Kd (Modified theories of gravity), 04.60.-m (Quantum gravity), 11.10.Ef (Lagrangian and Hamiltonian approach), 98.80.Cq (Particle-theory and field-theory models of the early Universe)

\section{Introduction}

In the recent years modified theories of gravity have attracted a considerable amount of attention. These modifications of General Relativity (GR) aim to improve the behaviour of the theory either at high energies (renormalizability, early-time universe) or at large distances (cosmology, galaxies), and sometimes at both regimes (see [1, 2] for such an attempt), in order to achieve a better agreement with observational data or a more plausible theoretical framework. Recently the so-called Covariant Renormalizable Gravity (CRG) was proposed in Refs. [3 5]. For a review of CRG and its comparison with other modified gravities, one can see [6]. CRG aims to provide a power-counting renormalizable field theory of gravity that is covariant under spacetime diffeomorphism and possesses local Lorentz invariance at the fundamental level. Lorentz invariance of the graviton propagator of CRG is, however, broken dynamically at high energies. This is achieved by introducing an exotic fluid of unknown origin, which is coupled to spacetime in a rather complicated way. Hořava-Lifshitz (HL) gravity [7] — another power-counting

\footnotetext{
${ }^{1}$ Electronic address: masud.chaichian@helsinki.fi

${ }^{2}$ Electronic address: markku.oksanen@helsinki.fi

${ }^{3}$ Electronic address: anca.tureanu@helsinki.fi
} 
renormalizable field theory of gravity — is based on the idea that space and time scale differently at high energies,

$$
\boldsymbol{x} \rightarrow b \boldsymbol{x}, \quad t \rightarrow b^{z} t,
$$

with a dynamic critical exponent $z=1,2,3, \ldots$ This enables one to modify the ultraviolet behaviour of the graviton propagator to $|\boldsymbol{k}|^{-2 z}$, where $\boldsymbol{k}$ is the spatial momentum. In $D$ spatial dimensions, $z=D$ ensures the theory is power-counting renormalizable. Such spacetime admits a preferred foliation into spatial hypersurfaces, and hence the local Lorentz invariance is broken. Note that more general theories of such sort have been proposed in Ref. [2]. CRG achieves a similar ultraviolet behaviour of the graviton propagator as HL gravity, but without introducing explicitly Lorentz noninvariant terms into the action. However, we should note that the renormalizability of CRG, as well as of the HL model, is assumed only based on the power-counting arguments. There are several potential pathologies that could ruin the renormalizability of this theory, such as gradient instabilities, ghosts or strong coupling. Since a violation of Lorentz invariance has never been observed, one could argue that CRG is a more natural modification of GR than the explicitly Lorentz noninvariant ones, in particular HL gravity and its generalizations (see e.g. [1, [2, 8, 9]).

Hamiltonian formalism provides a powerful tool for the analysis of constrained systems such as gravity. For example, it has been shown that HL gravity can be physically consistent $(N>0)$ at high energies only if the projectability condition is imposed on the lapse function $N$ [10]: $N=N(t)$. A similar result has also been obtained for the more general modified $F(R)$ HL gravity [11]. In this paper we seek to understand CRG from the point of view of Hamiltonian analysis.

First we obtain the Arnowitt-Deser-Misner (ADM) representations of the two most interesting actions of CRG theory in Sec. 2 - one supposedly power-counting renormalizable and the other power-counting super-renormalizable. Since the actions turn out to contain time derivatives up to fourth order, we consider ways to simplify the actions via introduction of additional scalar fields and partial gauge fixing. In Sec. 3 we analyze the actions using Hamiltonian formalism. Conclusions and some further discussions are given in Sec. 4

\section{ADM representation of covariant renormalizable gravity}

\section{$2.1 \quad$ Action}

Let us review the action of the CRG theory proposed in [3 [5]. In 4-dimensional spacetime the action of the supposedly power-counting renormalizable gravity is (corresponds to $z=3$ )

$S_{g, 3}=\int d^{4} x \sqrt{-g}\left\{\frac{R}{2 \kappa^{2}}-\alpha\left(T^{\mu \nu} R_{\mu \nu}+\beta T R\right)\left(T^{\mu \nu} \nabla_{\mu} \nabla_{\nu}+\gamma T \nabla^{\rho} \nabla_{\rho}\right)\left(T^{\mu \nu} R_{\mu \nu}+\beta T R\right)\right\}$.

We also consider the supposedly power-counting super-renormalizable gravity with the action (corresponds to $z=4$ )

$$
S_{g, 4}=\int d^{4} x \sqrt{-g}\left\{\frac{R}{2 \kappa^{2}}-\alpha\left[\left(T^{\mu \nu} \nabla_{\mu} \nabla_{\nu}+\gamma T \nabla^{\rho} \nabla_{\rho}\right)\left(T^{\mu \nu} R_{\mu \nu}+\beta T R\right)\right]^{2}\right\} .
$$


Here $T_{\mu \nu}$ is the energy-momentum tensor of a perfect non-relativistic fluid with an equation of state parameter $w \neq 1 / 3,-1$. We denote $T=g^{\mu \nu} T_{\mu \nu}$. The actions for higher $z$ could be analyzed similarly as these two interesting representatives of the odd and even $z$ actions.

In Ref. [4] it was proposed that the required perfect fluid can be realized with a constrained scalar field $\phi$ with the action

$$
S_{\phi}=\int d^{4} x \sqrt{-g}\left\{-\lambda\left(\frac{1}{2} \partial_{\mu} \phi \partial^{\mu} \phi+U(\phi)\right)\right\},
$$

where $\lambda$ is a Lagrange multiplier field. The energy-momentum tensor $T_{\mu \nu}$ is defined to be that of an isolated scalar field in a potential $V(\phi)$,

$$
T_{\mu \nu}^{\phi}=\partial_{\mu} \phi \partial_{\nu} \phi-g_{\mu \nu}\left(\frac{1}{2} \partial_{\rho} \phi \partial^{\rho} \phi+V(\phi)\right)
$$

regardless of the form of the CRG action. The constraint implied by the action (2.3),

$$
\frac{1}{2} \partial_{\mu} \phi \partial^{\mu} \phi+U(\phi)=0
$$

will be imposed from the start. Thus the constraint (2.5), and consequently the action (2.3), vanish in the total action of CRG. According to the constraint (2.5), if we assume $U(\phi)>0$, the vector $\partial_{\mu} \phi$ is timelike. Then at least locally one can choose the direction of time to be parallel to $\partial_{\mu} \phi$, so that the constraint (2.5) yields

$$
\frac{1}{2}\left(\frac{d \phi}{d t}\right)^{2}=U(\phi) \text {. }
$$

Then on the flat background metric one obtains the energy density $\rho_{\phi}=U(\phi)+V(\phi)$, the pressure $p_{\phi}=U(\phi)-V(\phi)$ and the equation of state parameter $w=p_{\phi} / \rho_{\phi}$ associated with $T_{\mu \nu}^{\phi}$. For simplicity, it is assumed that $V(\phi)$ and $U(\phi)$ are constants:

$$
U(\phi)=U_{0}, \quad V(\phi)=V_{0} .
$$

Now the action (2.1) can be written as

$$
\begin{array}{r}
S_{g, 3}=\int d^{4} x \sqrt{-g}\left\{\frac{R}{2 \kappa^{2}}-\alpha\left(\partial^{\mu} \phi \partial^{\nu} \phi R_{\mu \nu}+U_{0} R\right)\left(\partial^{\mu} \phi \partial^{\nu} \phi \nabla_{\mu} \nabla_{\nu}+2 U_{0} \nabla^{\rho} \nabla_{\rho}\right)\right. \\
\left.\times\left(\partial^{\mu} \phi \partial^{\nu} \phi R_{\mu \nu}+U_{0} R\right)\right\} .
\end{array}
$$

Likewise, the action for (2.2) is written as

$$
S_{g, 4}=\int d^{4} x \sqrt{-g}\left\{\frac{R}{2 \kappa^{2}}-\alpha\left[\left(\partial^{\mu} \phi \partial^{\nu} \phi \nabla_{\mu} \nabla_{\nu}+2 U_{0} \nabla^{\rho} \nabla_{\rho}\right)\left(\partial^{\mu} \phi \partial^{\nu} \phi R_{\mu \nu}+U_{0} R\right)\right]^{2}\right\} .
$$

Here the constraint (2.5) has been used and the following parameters have been determined:

$$
\beta=-\frac{w-1}{2(3 w-1)}=\frac{V_{0}}{2 U_{0}-4 V_{0}}, \quad \gamma=\frac{1}{3 w-1}=\frac{U_{0}-V_{0}}{2 U_{0}-4 V_{0}} .
$$

In order to eliminate $U_{0}$ from the actions (2.8) and (2.9) we can use the constraint (2.5) to write [5]

$$
\begin{gathered}
\partial^{\mu} \phi \partial^{\nu} \phi R_{\mu \nu}+U_{0} R=\partial^{\mu} \phi \partial^{\nu} \phi\left(R_{\mu \nu}-\frac{1}{2} g_{\mu \nu} R\right) \\
\partial^{\mu} \phi \partial^{\nu} \phi \nabla_{\mu} \nabla_{\nu}+2 U_{0} \nabla^{\rho} \nabla_{\rho}=\partial^{\mu} \phi \partial^{\nu} \phi\left(\nabla_{\mu} \nabla_{\nu}-g_{\mu \nu} \nabla^{\rho} \nabla_{\rho}\right) .
\end{gathered}
$$


Since (2.3) vanishes, the total action $\left(S_{z}=S_{g, z}+S_{\phi}\right)$ for (2.8) can be written

$$
\begin{aligned}
S_{3}=\int d^{4} x \sqrt{-g}\left\{\frac{R}{2 \kappa^{2}}-\alpha \partial^{\mu} \phi \partial^{\nu} \phi\left(R_{\mu \nu}-\frac{1}{2} g_{\mu \nu} R\right)\right. \\
\left.\quad \times \partial^{\mu} \phi \partial^{\nu} \phi\left(\nabla_{\mu} \nabla_{\nu}-g_{\mu \nu} \nabla^{\rho} \nabla_{\rho}\right) \partial^{\sigma} \phi \partial^{\lambda} \phi\left(R_{\sigma \lambda}-\frac{1}{2} g_{\sigma \lambda} R\right)\right\} .
\end{aligned}
$$

For (2.9) the total action can be written

$$
S_{4}=\int d^{4} x \sqrt{-g}\left\{\frac{R}{2 \kappa^{2}}-\alpha\left[\partial^{\mu} \phi \partial^{\nu} \phi\left(\nabla_{\mu} \nabla_{\nu}-g_{\mu \nu} \nabla^{\rho} \nabla_{\rho}\right) \partial^{\sigma} \phi \partial^{\lambda} \phi\left(R_{\sigma \lambda}-\frac{1}{2} g_{\sigma \lambda} R\right)\right]^{2}\right\}
$$

The corresponding Lagrangians are denoted by $L_{z}$, such that $S_{z}=\int d t L_{z}$.

\subsection{Space-time decomposition}

We consider the ADM decomposition of the gravitational field [12] (for reviews and mathematical background, see [13, 14]). Assume that the spacetime admits a foliation into $t=$ constant hypersurfaces $\Sigma_{t}$, whose future-directed unit normal is denoted by $n$. The metric tensor ${ }^{(4)} g_{a b}$ of spacetime is decomposed with respect to $\Sigma_{t}$ :

$$
\begin{array}{lll}
g_{a b}={ }^{(4)} g_{a b}-\epsilon n_{a} n_{b}, & n_{a} n^{a}=\epsilon, & t^{a} \nabla_{a} t=1, \\
N=\epsilon n_{a} t^{a}, & N^{a}=g^{a}{ }_{b} t^{b}, & t^{a}=N n^{a}+N^{a},
\end{array}
$$

where $\epsilon=-1(\epsilon=1)$ for a spacelike (timelike) $\Sigma_{t}$, and the orthogonal projector from $T \mathcal{M}$ to $T \Sigma_{t}$ is

$$
g_{b}^{a}=\delta_{b}^{a}-\epsilon n^{a} n_{b} .
$$

The extension of tensors on $\Sigma_{t}$ to tensors on $\mathcal{M}$ is induced by the embedding of $\Sigma_{t}$ to $\mathcal{M}$, and by the projector map (2.16). Such extended quantities are, for example, the metric tensor $g_{a b}$ of $\Sigma_{t}$ and the shift vector $N^{a}$. From now on quantities defined on the spacetime $\mathcal{M}$ and associated with its metric ${ }^{(4)} g_{a b}$ are marked with the prefix ${ }^{(4)}$. We denote the covariant derivatives on $\mathcal{M}$ and $\Sigma_{t}$ by $\nabla$ and $D$, respectively.

Taking the scalar field $t$ as the time coordinate, when $t^{a}$ is the "flow of time" vector, and assuming $\Sigma_{t}$ is spacelike $(\epsilon=-1)$, we can write the unit normal in terms of the lapse $N$ and the shift vector $N^{i}$ :

$$
n_{\mu}=-N \nabla_{\mu} t=(-N, 0,0,0), \quad n^{\mu}=\left(n^{0}, n^{i}\right)=\left(\frac{1}{N},-\frac{N^{i}}{N}\right) .
$$

The components of the metric of spacetime are decomposed

$$
{ }^{(4)} g_{00}=-N^{2}+N_{i} N^{i}, \quad{ }^{(4)} g_{0 i}={ }^{(4)} g_{i 0}=N_{i}, \quad{ }^{(4)} g_{i j}=g_{i j}
$$

and the components of its inverse are

$$
{ }^{(4)} g^{00}=-1 / N^{2}, \quad{ }^{(4)} g^{0 i}={ }^{(4)} g^{i 0}=N^{i} / N^{2}, \quad{ }^{(4)} g^{i j}=g^{i j}-\left(N^{i} N^{j} / N^{2}\right) .
$$

The natural volume element decomposes

$$
d^{4} x \sqrt{-{ }^{(4)} g}=d t d^{3} \boldsymbol{x} \sqrt{g} N .
$$


We assume $c=1$ units.

A 4 -vector $u^{\mu}$ can be decomposed into components perpendicular and parallel to $n^{\mu}$ :

$$
u^{\mu}={ }_{\llcorner} u^{\mu}+\epsilon(n \cdot u) n^{\mu}, \quad{ }_{\perp} u^{\mu} \equiv g_{\nu}^{\mu} u^{\nu}=u^{\mu}-\epsilon(n \cdot u) n^{\mu} .
$$

For $u^{\mu}=\partial^{\mu} \phi=\nabla^{\mu} \phi$ by using (2.17) we get

$$
{ }_{\perp} u^{\mu}={ }_{\perp} \nabla^{\mu} \phi=\left(0, D^{i} \phi\right), \quad D^{i} \phi \equiv g^{i j} D_{j} \phi=g^{i j} \partial_{j} \phi,
$$

and

$$
n \cdot u=\nabla_{n} \phi \equiv n^{\mu} \nabla_{\mu} \phi .
$$

Note that for a vector ${ }_{\perp} u^{\mu} \in T \Sigma_{t}$ and a covector ${ }_{\perp} v_{\mu} \in T^{*} \Sigma_{t}$ we have the zero-components

$$
{ }_{\llcorner} u^{0}=0, \quad{ }^{0} v_{0}=N^{i}{ }_{\perp} v_{i} .
$$

This generalizes straightforwardly for tensors of any type defined on $\Sigma_{t}$.

We will apply the ADM decomposition to the actions (2.13) and (2.14). The scalar curvature ${ }^{(4)} R$ of spacetime decomposes as usual:

$$
\begin{aligned}
{ }^{(4)} R & =K_{i j} K^{i j}-K^{2}+R+2 \nabla_{\mu}\left(n^{\mu} \nabla_{\nu} n^{\nu}-n^{\nu} \nabla_{\nu} n^{\mu}\right) \\
& =K_{i j} K^{i j}-K^{2}+R+2 \nabla_{\mu}\left(n^{\mu} K\right)-\frac{2}{N} D^{i} D_{i} N .
\end{aligned}
$$

Here $R$ is the intrinsic scalar curvature of $\Sigma_{t}$ and $K_{i j}$ is the extrinsic curvature of $\Sigma_{t}$ :

$$
K_{i j}=\frac{1}{2 N}\left(\dot{g}_{i j}-2 D_{(i} N_{j)}\right), \quad K=g^{i j} K_{i j}
$$

where the dot denotes the derivative with respect to time $t$.

The covariant derivative of the normal covector $n_{\mu}$ can be written

$$
\nabla_{\mu} n_{\nu}=K_{\mu \nu}-n_{\mu} D_{\nu} \ln N
$$

and it implies the following relations:

$$
\nabla_{n} n_{\mu}=D_{\mu} \ln N, \quad n^{\nu} \nabla_{\mu} n_{\nu}=0, \quad \nabla_{\mu} n^{\mu}=K .
$$

These relations have been used in (2.25). Note that we can also write

$$
\nabla_{\mu}\left(n^{\mu} K\right)=K^{2}+\nabla_{n} K
$$

for example, in (2.25).

The Ricci tensor ${ }^{(4)} R_{\mu \nu}$ of spacetime can be decomposed as

$$
{ }^{(4)} R_{\mu \nu}={ }_{\perp}^{(4)} R_{\mu \nu}-{ }_{\perp}^{(4)} R_{\mu n} n_{\nu}-{ }_{\perp}^{(4)} R_{n \nu} n_{\mu}+{ }^{(4)} R_{n n} n_{\mu} n_{\nu},
$$

where we have defined

$$
\begin{aligned}
{ }_{\perp}^{(4)} R_{\mu \nu} & \equiv g^{\rho}{ }_{\mu} g^{\sigma}{ }_{\nu}^{(4)} R_{\rho \sigma}=R_{\mu \nu}+K K_{\mu \nu}-2 K_{\mu \rho} K^{\rho}{ }_{\nu}-\frac{1}{N} D_{\mu} D_{\nu} N+\frac{1}{N} \mathcal{L}_{N n} K_{\mu \nu}, \\
{ }_{\perp}^{(4)} R_{\mu n} & \equiv g^{\rho}{ }_{\mu} n^{\nu(4)} R_{\rho \nu}=D_{\rho} K^{\rho}{ }_{\mu}-D_{\mu} K \\
{ }^{(4)} R_{n \nu} & \equiv g^{\rho}{ }_{\nu} n^{\mu(4)} R_{\mu \rho}=D_{\rho} K^{\rho}{ }_{\nu}-D_{\nu} K \\
{ }^{(4)} R_{n n} & \equiv{ }^{(4)} R_{\mu \nu} n^{\mu} n^{\nu}=\frac{1}{2}\left(K^{2}-K_{i j} K^{i j}+R-{ }^{(4)} R\right) \\
& =K^{2}-K_{i j} K^{i j}-\nabla_{\mu}\left(n^{\mu} K\right)+\frac{1}{N} D^{i} D_{i} N .
\end{aligned}
$$


In Eq. (2.31) $R_{\mu \nu}$ is the Ricci tensor of the hypersurface $\Sigma_{t}$ and $\mathcal{L}_{N n}$ denotes the Lie derivative along $N n^{\mu}$. Note that for any tensor field $T$ that is tangent to $\Sigma_{t}, \mathcal{L}_{N n} T$ is also tangent to $\Sigma_{t}$. In Eqs. (2.31) -(2.34) we have used the Gauss relation, the Ricci equation and the Codazzi relation, and in (2.34) the decomposition of ${ }^{(4)} R$ from (2.25) was also used. Hence the Einstein tensor can be decomposed:

$$
\begin{gathered}
{ }^{(4)} G_{\mu \nu} \equiv{ }^{(4)} R_{\mu \nu}- \\
\frac{1}{2}{ }^{(4)} g_{\mu \nu}{ }^{(4)} R=R_{\mu \nu}+K K_{\mu \nu}-2 K_{\mu \rho} K_{\nu}^{\rho}-\frac{1}{N} D_{\mu} D_{\nu} N+\frac{1}{N} \mathcal{L}_{N n} K_{\mu \nu} \\
-\frac{1}{2} g_{\mu \nu}\left(R+K_{i j} K^{i j}+K^{2}+2 \nabla_{n} K-\frac{2}{N} D^{i} D_{i} N\right) \\
+\left(D_{\mu} K-D_{\rho} K^{\rho}{ }_{\mu}\right) n_{\nu}+n_{\mu}\left(D_{\nu} K-D_{\rho} K^{\rho}{ }_{\nu}\right)+\frac{1}{2} n_{\mu} n_{\nu}\left(K^{2}-K_{i j} K^{i j}+R\right),
\end{gathered}
$$

where we have also used (2.29). Thus in the actions (2.13) and (2.14) we have

$$
\begin{aligned}
\partial^{\mu} \phi \partial^{\nu}{ }^{(4)} G_{\mu \nu}= & D^{i} \phi D^{j} \phi\left[R_{i j}+K K_{i j}-2 K_{i k} K^{k}{ }_{j}-\frac{1}{N} D_{i} D_{j} N+\frac{1}{N} \mathcal{L}_{N n} K_{i j}\right. \\
& \left.-\frac{1}{2} g_{i j}\left(R+K_{k l} K^{k l}+K^{2}+2 \nabla_{n} K-\frac{2}{N} D^{k} D_{k} N\right)\right] \\
& +2\left(\nabla_{n} \phi\right) D^{i} \phi\left(D_{i} K-D^{j} K_{j i}\right)+\frac{1}{2}\left(\nabla_{n} \phi\right)^{2}\left(K^{2}-K_{i j} K^{i j}+R\right) .
\end{aligned}
$$

Let us then consider the covariant derivatives in the actions (2.13) and (2.14). The covariant derivative $D$ of a $(k, l)$-tensor field $T$ on $\Sigma_{t}$ is given by

$$
D_{\mu} T_{\rho_{1} \cdots \rho_{l}}^{\nu_{1} \cdots \nu_{k}}=g_{\mu}^{\sigma} g_{\alpha_{1}}^{\nu_{1}} \cdots g_{\alpha_{k}}^{\nu_{k}} g_{\rho_{1}}^{\beta_{1}} \cdots g_{\rho_{l}}^{\beta_{l}} \nabla_{\sigma} T_{\beta_{1} \cdots \beta_{l}}^{\alpha_{1} \cdots \alpha_{k}}
$$

where in the right-hand side one considers the extension of $T$ on $\mathcal{M}$. For a scalar field $f$, $D_{\mu} f=g_{\mu}^{\nu} \nabla_{\nu} f$ implies the decomposition

$$
\nabla_{\mu} f=D_{\mu} f-n_{\mu} \nabla_{n} f
$$

For the second order covariant derivative we obtain

$$
D_{\mu} D_{\nu} f=g_{\mu}^{\rho} g_{\nu}^{\sigma} \nabla_{\rho} \nabla_{\sigma} f+g_{(\mu}^{\rho} g_{\nu)}^{\sigma}\left(\nabla_{\rho} g_{\sigma}^{\lambda}\right) \nabla_{\lambda} f
$$

Note that both $D$ and $\nabla$ are torsion-free, which is the reason why the last term is symmetrized over $\mu$ and $\nu$. Introducing (2.16) into (2.39) yields

$$
\begin{aligned}
\nabla_{\mu} \nabla_{\nu} f=D_{\mu} D_{\nu} f- & 2 n_{(\mu \mid} \nabla_{n} \nabla_{\mid \nu)} f-n_{\mu} n_{\nu} n^{\rho} n^{\sigma} \nabla_{\rho} \nabla_{\sigma} f \\
& -\left(\nabla_{(\mu} n_{\nu)}+n_{(\mu \mid} \nabla_{n} n_{\mid \nu)}+n_{(\mu} n^{\rho} \nabla_{\nu)} n_{\rho}+n_{\mu} n_{\nu} n^{\rho} \nabla_{n} n_{\rho}\right) \nabla_{n} f .
\end{aligned}
$$

Then we shall use the relations (2.27) and (2.28) to obtain

$$
\nabla_{\mu} \nabla_{\nu} f=D_{\mu} D_{\nu} f-K_{\mu \nu} \nabla_{n} f-2 n_{(\mu} g_{\nu)}^{\rho} \nabla_{n} \nabla_{\rho} f+n_{\mu} n_{\nu} n^{\rho} n^{\sigma} \nabla_{\rho} \nabla_{\sigma} f
$$

As the last step in the decomposition we write

$$
\nabla_{n} \nabla_{\rho} f=\nabla_{\rho} \nabla_{n} f-\nabla_{\rho} n^{\sigma} \nabla_{\sigma} f=\nabla_{\rho} \nabla_{n} f-\left(K_{\rho}^{\sigma}-n_{\rho} D^{\sigma} \ln N\right) \nabla_{\sigma} f
$$


which implies

$$
\begin{aligned}
g^{\rho}{ }_{\nu} \nabla_{n} \nabla_{\rho} f & =D_{\nu} \nabla_{n} f-K_{\nu}{ }^{\rho} \nabla_{\rho} f \\
n^{\rho} n^{\sigma} \nabla_{\rho} \nabla_{\sigma} f & =\nabla_{n} \nabla_{n} f-\left(D^{i} \ln N\right) D_{i} f .
\end{aligned}
$$

Thus the final decomposition is

$$
\begin{aligned}
\nabla_{\mu} \nabla_{\nu} f=D_{\mu} D_{\nu} f-K_{\mu \nu} \nabla_{n} f-2 n_{(\mu} D_{\nu)} \nabla_{n} f & -2 n_{(\mu} K_{\nu)}{ }^{\rho} \nabla_{\rho} f \\
& +n_{\mu} n_{\nu}\left(\nabla_{n} \nabla_{n} f-\left(D^{i} \ln N\right) D_{i} f\right)
\end{aligned}
$$

The contraction of (2.45) with ${ }^{(4)} g^{\mu \nu}$ gives

$$
\nabla^{\mu} \nabla_{\mu} f=D^{i} D_{i} f-K \nabla_{n} f-\nabla_{n} \nabla_{n} f+\left(D^{i} \ln N\right) D_{i} f,
$$

where we have also used

$$
{ }^{(4)} g^{\mu \nu} D_{\mu} D_{\nu} f=g^{i j} D_{i} D_{j} f=D^{i} D_{i} f, \quad{ }^{(4)} g^{\mu \nu} K_{\mu \nu}=g^{i j} K_{i j}=K .
$$

Then in the action we have

$$
\begin{gathered}
\partial^{\mu} \phi \partial^{\nu} \phi\left(\nabla_{\mu} \nabla_{\nu}-{ }^{(4)} g_{\mu \nu} \nabla^{\rho} \nabla_{\rho}\right) f=D^{i} \phi D^{j} \phi\left[\left(D_{i} D_{j}-g_{i j} D^{k} D_{k}-g_{i j}\left(D^{k} \ln N\right) D_{k}\right) f\right. \\
\left.-\left(K_{i j}-g_{i j} K\right) \nabla_{n} f+g_{i j} \nabla_{n} \nabla_{n} f\right] \\
-2\left(\nabla_{n} \phi\right) D^{i} \phi\left(D_{i} \nabla_{n}+K_{i j} D^{j}\right) f+\left(\nabla_{n} \phi\right)^{2}\left(D^{i} D_{i}-K \nabla_{n}\right) f .
\end{gathered}
$$

The covariant derivative of a scalar $f$ along the normal $n$ is given by

$$
\nabla_{n} f=\frac{1}{N}\left(\dot{f}-N^{i} \partial_{i} f\right)
$$

Now we have everything that is needed for the ADM decomposition of the actions of CRG. First, in order to shorten the expressions, we denote the decomposition (2.36) by

$$
{ }^{(4)} \mathcal{G}_{\phi} \equiv \partial^{\mu} \phi \partial^{\nu} \phi^{(4)} G_{\mu \nu}
$$

Then the action (2.13) is written in terms of the ADM variables:

$$
\begin{aligned}
S_{3}=\int d t d^{3} \boldsymbol{x} \sqrt{g} N\left\{\frac{K_{i j} K^{i j}-K^{2}+R}{2 \kappa^{2}}-\alpha^{(4)} \mathcal{G}_{\phi}\left[D ^ { i } \phi D ^ { j } \phi \left[\left(D_{i} D_{j}-g_{i j} D^{k} D_{k}\right.\right.\right.\right. \\
\left.\left.-g_{i j}\left(D^{k} \ln N\right) D_{k}\right)^{(4)} \mathcal{G}_{\phi}-\left(K_{i j}-g_{i j} K\right) \nabla_{n}{ }^{(4)} \mathcal{G}_{\phi}+g_{i j} \nabla_{n} \nabla_{n}{ }^{(4)} \mathcal{G}_{\phi}\right] \\
\left.\left.-2\left(\nabla_{n} \phi\right) D^{i} \phi\left(D_{i} \nabla_{n}+K_{i j} D^{j}\right)^{(4)} \mathcal{G}_{\phi}+\left(\nabla_{n} \phi\right)^{2}\left(D^{i} D_{i}-K \nabla_{n}\right)^{(4)} \mathcal{G}_{\phi}\right]\right\} .
\end{aligned}
$$

The action (2.14) can be written as

$$
\begin{aligned}
S_{4}=\int d t d^{3} \boldsymbol{x} \sqrt{g} N\left\{\frac{K_{i j} K^{i j}-K^{2}+R}{2 \kappa^{2}}-\alpha\left[D ^ { i } \phi D ^ { j } \phi \left[\left(D_{i} D_{j}-g_{i j} D^{k} D_{k}\right.\right.\right.\right. \\
\left.\left.\quad-g_{i j}\left(D^{k} \ln N\right) D_{k}\right)^{(4)} \mathcal{G}_{\phi}-\left(K_{i j}-g_{i j} K\right) \nabla_{n}{ }^{(4)} \mathcal{G}_{\phi}+g_{i j} \nabla_{n} \nabla_{n}{ }^{(4)} \mathcal{G}_{\phi}\right] \\
\left.\left.-2\left(\nabla_{n} \phi\right) D^{i} \phi\left(D_{i} \nabla_{n}+K_{i j} D^{j}\right)^{(4)} \mathcal{G}_{\phi}+\left(\nabla_{n} \phi\right)^{2}\left(D^{i} D_{i}-K \nabla_{n}\right)^{(4)} \mathcal{G}_{\phi}\right]^{2}\right\} .
\end{aligned}
$$

We assume that the boundary terms originating from total derivatives vanish when appropriate boundary conditions are imposed, even though the role of surface integrals is 
known to be very important in general relativity. Introducing the decomposition (2.36) and the covariant derivative along $n$ (2.49) explicitly into (2.51) would clearly result into a very complicated expression. The action (2.51) contains time derivatives of the metric $g_{i j}$ up to fourth order, time derivatives of the lapse $N$ and the shift vector $N^{i}$ (or of its spatial derivative $D_{i} N_{j}$ ) up to third order, and time derivatives of $\phi$ up to third order. Some higher time derivatives of the ADM variables could be removed via integration by parts, but with the price of introducing even higher time derivatives of the scalar field $\phi$.

For the Lagrangian $L_{3}$ defined by the action (2.51) we find

$$
\frac{\delta^{2} L_{3}}{\delta g_{i j}^{(4)} \delta g_{k l}^{(4)}}=0
$$

where $g_{i j}^{(n)} \equiv \frac{\partial^{n} g_{i j}}{\partial t^{n}}$. This is clear since the highest time derivative is contained in the term

$$
\alpha^{(4)} \mathcal{G}_{\phi}\left(D^{i} \phi D_{i} \phi\right)^{2} \nabla_{n} \nabla_{n} \nabla_{n} K
$$

where

$$
\nabla_{n} \nabla_{n} \nabla_{n} K=\frac{g^{i j}}{2 N^{4}} g_{i j}^{(4)}+(\text { terms with lower order time derivatives }) .
$$

Thus $g_{i j}^{(4)}$ cannot be solved in terms of the canonical variables, nor $g_{i j}^{(8)}$ defined in terms of the initial data from the equations of motion.

For the Lagrangian $L_{4}$ defined by the action (2.52) the highest time derivative is contained in the term

$$
-\alpha\left(D^{i} \phi D_{i} \phi\right)^{3}\left(\nabla_{n} \nabla_{n} \nabla_{n} K\right)^{2}
$$

and

$$
\frac{\delta^{2} L_{4}}{\delta g_{i j}^{(4)} \delta g_{k l}^{(4)}}=-\sqrt{g} N \frac{g^{i j} g^{k l}}{4 N^{8}} \alpha\left(D^{m} \phi D_{m} \phi\right)^{3} .
$$

Therefore we expect that $g_{i j}^{(4)}$ could be solved in terms of the canonical variables.

However, since neither of the actions (2.51) or (2.52) depend on $N^{(4)}, N^{i(4)}$ or $\phi^{(4)}$, the highest order Hessian matrices

$$
\frac{\delta^{2} L_{3}}{\delta q_{I}^{(4)} \delta q_{J}^{(4)}} \quad \text { and } \quad \frac{\delta^{2} L_{4}}{\delta q_{I}^{(4)} \delta q_{J}^{(4)}}
$$

where $q_{I}=\left(\phi, N, N^{i}, g_{i j}\right), I=1,2, \ldots, 11$, have the ranks zero and six everywhere, respectively. Thus there are eleven and five Lagrangian constraints associated with $L_{3}$ and $L_{4}$, respectively. As expected, both Lagrangians are singular. The reason for the singularity is the presence of gauge symmetry, and its associated (first-class) constraints.

The actions proposed in Refs. [4, 5] for higher $z(z>4)$ can also be written in the ADM form by using the decompositions we have obtained above, (2.36) and (2.48) in particular. Then we see that for the Lagrangian of even $z=2 n+2$ and of odd $z=2 n+3$ the highest order Hessian matrix is singular, similarly to the two cases discussed above. 


\subsection{Partial gauge fixing}

The constraint (2.5) on $\phi$ ensures that the vector $\partial^{\mu} \phi$ is timelike everywhere, when $U(\phi)>0$ is assumed. Therefore there exists a preferred foliation of space-time into spatial hypersurfaces $\Sigma_{t}$ whose unit normal is given by

$$
n^{\mu}=-\frac{\partial^{\mu} \phi}{\sqrt{-\partial_{\nu} \phi \partial^{\nu} \phi}}=-\frac{\partial^{\mu} \phi}{\sqrt{2 U(\phi)}} .
$$

Hence we can write

$$
\partial_{\mu} \phi=-\sqrt{2 U(\phi)} n_{\mu}, \quad \partial^{\mu} \phi=-\sqrt{2 U(\phi)} n^{\mu},
$$

where the unit normal is given in terms of the ADM variables in Eq. (2.17). From Eq. (2.60) we see that in this foliation $\phi$ is constant on $\Sigma_{t}, \phi=\phi(t)$. Thus the constraint (2.5) on $\phi$ reduces to

$$
-\frac{\dot{\phi}^{2}}{2 N^{2}}+U(\phi)=0
$$

This implies that the lapse $N$ must be constant on $\Sigma_{t}$ too, $N=N(t)$. In order to preserve this condition we restrict the symmetry under diffeomorphisms of space-time to the symmetry under foliation-preserving diffeomorphisms, given in the infinitesimal form as

$$
\delta t=f(t), \quad \delta \boldsymbol{x}=\boldsymbol{\xi}(t, \boldsymbol{x}) .
$$

This is the main symmetry group of the HL gravity [7]. In the language of Horava's theory we would say that both $\phi$ and $N$ are projectable - like the lapse $N$ is in projectable HL gravity. Here we consider (2.62) as a partial gauge fixing of the diffeomorphism symmetry.

Now the Eqs. (2.36) and (2.48) can be written

$$
\begin{gathered}
\partial^{\mu} \phi \partial^{\nu} \phi^{(4)} G_{\mu \nu}=U_{0}\left(K^{2}-K_{i j} K^{i j}+R\right) \\
\partial^{\mu} \phi \partial^{\nu} \phi\left(\nabla_{\mu} \nabla_{\nu}-{ }^{(4)} g_{\mu \nu} \nabla^{\rho} \nabla_{\rho}\right) f=2 U_{0}\left(D^{i} D_{i}-K \nabla_{n}\right) f,
\end{gathered}
$$

where (2.7) has again been assumed. Note that $\phi$ is no longer an independent variable, since the constraint (2.61) implies

$$
\phi(t)=\phi\left(t_{0}\right)+\sqrt{2 U_{0}} \int_{t_{0}}^{t} d t^{\prime} N\left(t^{\prime}\right) .
$$

Moreover, $\phi$ is not even present in the actions anymore, as we will see next. The actions (2.51) and (2.52) reduce to

$$
\begin{aligned}
S_{3}=\int d t d^{3} \boldsymbol{x} \sqrt{g} N\left\{\frac{K_{i j} K^{i j}-K^{2}+R}{2 \kappa^{2}}\right. & -\alpha 2 U_{0}^{3}\left(K^{2}-K_{i j} K^{i j}+R\right) \\
& \left.\times\left(D^{i} D_{i}-K \nabla_{n}\right)\left(K^{2}-K_{i j} K^{i j}+R\right)\right\}
\end{aligned}
$$

and

$$
\begin{aligned}
S_{4}=\int d t d^{3} \boldsymbol{x} \sqrt{g} N\left\{\frac{K_{i j} K^{i j}-K^{2}+R}{2 \kappa^{2}}\right. & \\
& \left.-\alpha\left[2 U_{0}^{2}\left(D^{i} D_{i}-K \nabla_{n}\right)\left(K^{2}-K_{i j} K^{i j}+R\right)\right]^{2}\right\} .
\end{aligned}
$$


Although these actions have been simplified considerably, they still contain second order time derivatives of the metric $g_{i j}$, and first order time derivatives of the lapse $N$ and the shift vector $N^{i}$. Indeed, we obtain

$$
\begin{array}{r}
\nabla_{n}\left(K^{2}-K_{i j} K^{i j}+R\right)=\frac{1}{N}\left[2\left(\dot{K}-N^{i} \partial_{i} K\right) K-2\left(\dot{K}_{i j}-N^{k} \partial_{k} K_{i j}\right) K^{i j}\right. \\
\left.-2\left(\dot{g}^{i j}-N^{k} \partial_{k} g^{i j}\right) K_{i l} K_{j}{ }^{l}+\left(\dot{R}-N^{i} \partial_{i} R\right)\right]
\end{array}
$$

where the time derivatives of the extrinsic curvature (2.26) and the inverse metric $g^{i j}$ are given by

$$
\begin{gathered}
\dot{K}_{i j}=-\frac{\dot{N}}{N} K_{i j}+\frac{1}{2 N}\left(\ddot{g}_{i j}-2 \partial_{t} D_{(i} N_{j)}\right), \\
\dot{K}=\dot{g}^{i j} K_{i j}+g^{i j} \dot{K}_{i j}, \quad \dot{g}^{i j}=-g^{i k} g^{j l} \dot{g}_{k l} .
\end{gathered}
$$

We can see that our earlier observations on the singularity of $L_{3}$ and $L_{4}$ have not changed. For the Lagrangian $L_{3}$ we obtain

$$
\frac{\delta^{2} L_{3}}{\delta \ddot{g}_{i j} \delta \ddot{g}_{k l}}=0 .
$$

For the Lagrangian $L_{4}$ we obtain

$$
\frac{\delta^{2} L_{4}}{\delta \ddot{g}_{i j} \delta \ddot{g}_{k l}}=-\sqrt{g} \alpha \frac{8 U_{0}^{4}}{N^{3}} K^{2}\left(K^{i j}-g^{i j} K\right)\left(K^{k l}-g^{k l} K\right) .
$$

Thus for the Hessian matrices we find

$$
\operatorname{rank}\left[\frac{\delta^{2} L_{3}}{\delta \ddot{q}_{I} \delta \ddot{q}_{J}}\right]=0, \quad \operatorname{rank}\left[\frac{\delta^{2} L_{4}}{\delta \ddot{q}_{I} \delta \ddot{q}_{J}}\right]=6,
$$

and hence we expect ten and four Lagrangian constraints for $L_{3}$ and $L_{4}$, respectively.

Second order time derivatives in the Lagrangian of a theory of gravity are not necessarily a fatal problem. Indeed we know that actions of the type $f\left({ }^{(4)} R\right)$ do not suffer from the Ostrogradskian instability (for a proper explanation, see the discussion in Ref. [25]), though the scalar curvature (2.25) contains the second order time derivative term

$$
\left.2 \nabla_{\mu}\left(n^{\mu} K\right)=\frac{g^{i j} \ddot{g}_{i j}}{N^{2}}+\text { (terms with first order time derivatives }\right) .
$$

In the $f\left({ }^{(4)} R\right)$ action one can get rid of the second order time derivative by introducing additional scalar fields and integrating by parts, although with the price of an extra dynamical scalar degree of freedom - the scalaron. In the present case a somewhat

similar approach can reduce the number of time derivatives in the action. This will be discussed next.

\subsection{Further reducing the number of time derivatives}

As discussed in the Appendix of Ref. [5], in order to reduce the number of derivatives in the action, one can introduce four scalar fields $\zeta_{1}, \xi_{1}, \zeta_{2}$ and $\xi_{2}$, and write the action (2.13) in the form [5]:

$$
\begin{array}{r}
S_{3}=\int d^{4} x \sqrt{-{ }^{(4)} g}\left\{\frac{{ }^{(4)} R}{2 \kappa^{2}}-\alpha\left[\zeta_{1} \zeta_{2}+\xi_{1}\left(\zeta_{1}-\partial^{\mu} \phi \partial^{\nu} \phi\left(\nabla_{\mu} \nabla_{\nu}-{ }^{(4)} g_{\mu \nu} \nabla^{\rho} \nabla_{\rho}\right) \zeta_{2}\right)\right.\right. \\
\left.\left.+\xi_{2}\left(\zeta_{2}-\partial^{\mu} \phi \partial^{\nu} \phi^{(4)} G_{\mu \nu}\right)\right]\right\} .
\end{array}
$$


For the action (2.14) we introduce three scalar field $\eta, \zeta, \xi$ in order to write:

$$
\begin{aligned}
& S_{4}=\int d^{4} x \sqrt{-{ }^{(4)} g}\left\{\frac{{ }^{(4)} R}{2 \kappa^{2}}+\alpha\left[\eta^{2}-2 \eta \partial^{\mu} \phi \partial^{\nu} \phi\left(\nabla_{\mu} \nabla_{\nu}-{ }^{(4)} g_{\mu \nu} \nabla^{\rho} \nabla_{\rho}\right) \zeta\right.\right. \\
&\left.\left.+\xi\left(\zeta-\partial^{\mu} \phi \partial^{\nu} \phi^{(4)} G_{\mu \nu}\right)\right]\right\} .
\end{aligned}
$$

The bad news is that the actions (2.75) and (2.76) still contain second order time derivatives of the scalar fields $\zeta_{2}$ and $\zeta$, respectively.

However, if we employ the preferred foliation discussed in Sec. 2.3, we obtain actions with only first order derivatives with respect to time. Moreover, the kinetic terms of the actions are quadratic in the first time derivatives of the fields. Indeed, we obtain the following ADM representations of the actions (2.75) and (2.76):

$$
\begin{array}{r}
S_{3}=\int d t d^{3} \boldsymbol{x} \sqrt{g} N\left\{\frac{\mathcal{G}^{i j k l} K_{i j} K_{k l}+R}{2 \kappa^{2}}-\alpha\left[\zeta_{1} \zeta_{2}+\xi_{1}\left(\zeta_{1}+2 U_{0}\left(K \nabla_{n}-D^{i} D_{i}\right) \zeta_{2}\right)\right.\right. \\
\left.\left.+\xi_{2}\left(\zeta_{2}+U_{0}\left(\mathcal{G}^{i j k l} K_{i j} K_{k l}-R\right)\right)\right]\right\}
\end{array}
$$

and

$$
\begin{aligned}
& S_{4}=\int d t d^{3} \boldsymbol{x} \sqrt{g} N\left\{\frac{\mathcal{G}^{i j k l} K_{i j} K_{k l}+R}{2 \kappa^{2}}+\alpha\left[\eta^{2}+4 U_{0} \eta\left(K \nabla_{n}-D^{i} D_{i}\right) \zeta\right.\right. \\
& \left.\left.+\xi\left(\zeta+U_{0}\left(\mathcal{G}^{i j k l} K_{i j} K_{k l}-R\right)\right)\right]\right\} .
\end{aligned}
$$

Here we have introduced the so-called De Witt metric

$$
\mathcal{G}^{i j k l}=\frac{1}{2}\left(g^{i k} g^{j l}+g^{i l} g^{j k}\right)-g^{i j} g^{k l},
$$

which is the special case $\sigma=1$ of the "generalized De Witt metric"

$$
\mathcal{G}_{(\sigma)}^{i j k l}=\frac{1}{2}\left(g^{i k} g^{j l}+g^{i l} g^{j k}\right)-\sigma g^{i j} g^{k l} .
$$

When $\sigma \neq 1 / 3$, (2.80) has the inverse

$$
\mathcal{G}_{(\sigma) i j k l}=\frac{1}{2}\left(g_{i k} g_{j l}+g_{i l} g_{j k}\right)-\frac{\sigma}{3 \sigma-1} g_{i j} g_{k l}, \quad \mathcal{G}_{(\sigma) i j k l} \mathcal{G}_{(\sigma)}^{k l m n}=\delta_{(i}^{(m} \delta_{j)}^{n)} .
$$

Note that the actions (2.77) and (2.78) no longer contain time derivatives of the lapse $N$ and the shift vector $N^{i}$. This means that the lapse and the shift vector are nondynamical, and hence do not propagate, which is also an attractive feature. Obviously the Lagrangians of (2.77) and (2.78) are still singular due to the remaining gauge symmetry (2.62). The Hessian matrix for the Lagrangian $L_{3}$ of (2.77) is given by

$$
\frac{\delta^{2} L_{3}}{\delta \dot{g}_{i j} \delta \dot{g}_{k l}}=\sqrt{g}\left(\frac{1}{2 \kappa^{2}}-\alpha U_{0} \xi_{2}\right) \frac{1}{2 N} \mathcal{G}^{i j k l}, \quad \frac{\delta^{2} L_{3}}{\delta \dot{\zeta}_{2} \delta \dot{g}_{i j}}=-\sqrt{g} \alpha U_{0} \frac{g^{i j} \xi_{1}}{N}
$$

and the rest of the components are zero. The nonvanishing components of the Hessian matrix for the Lagrangian $L_{4}$ of (2.78) are

$$
\frac{\delta^{2} L_{4}}{\delta \dot{g}_{i j} \delta \dot{g}_{k l}}=\sqrt{g}\left(\frac{1}{2 \kappa^{2}}+\alpha U_{0} \xi\right) \frac{1}{2 N} \mathcal{G}^{i j k l}, \quad \frac{\delta^{2} L_{4}}{\delta \dot{\zeta} \delta \dot{g}_{i j}}=\sqrt{g} \alpha 2 U_{0} \frac{g^{i j} \eta}{N} .
$$

Thus we expect to have seven and six Lagrangian constraints for $L_{3}$ and $L_{4}$, respectively. 


\section{Hamiltonian formalism}

A Hamiltonian formalism for higher derivative theories was first developed by Ostrogradski [15]. Regular Lagrangians with higher order time derivatives are known to possess negative energy degrees of freedom that destabilize solutions of the theory [15, 16]. But gauge theories are never regular, so Dirac's formalism [17 19], which is suited for constrained (singular) systems, has to be used instead. Generalization of Dirac's formalism to higher derivative theories, and a generalization of Ostrogradski's formalism to singular systems, were finally achieved in Ref. [20].

Lagrangians with higher order time derivatives have also been studied in the context of nonlocal theories. In particular see [21], though only regular higher order Lagrangians are considered there, whereas all the CRG Lagrangians are singular. Theories where higher derivative terms are regarded as corrections to a corresponding lower order theory, and especially theories that are truncated perturbative expansions of a nonlocal theory, should employ so-called perturbative constraints that ensure that the higher order corrections do not change the theory qualitatively (e.g. introduce extra degrees of freedom, lack of lower energy bound or violation of unitarity) [16, 22].

GR with a minimal coupling to a scalar $\phi$ that is constrained by the same Lagrange multiplier constraint (2.5) as in CRG has been proposed in Ref. [23]. Its Hamiltonian formalism has been studied in Ref. [24].

The Hamiltonian analysis of the actions (2.51) and (2.52), which contain time derivatives up to fourth order, could be done using the formalism of Ref. [20]. Likewise, we could analyze the simpler actions (2.66) and (2.67) or (2.77) and (2.78), which contain second order time derivatives. Here we, however, concentrate on the analysis of the first order actions (2.77) and (2.78). The motivation is to gain some understanding from these simplified CRG actions, because the general ones have proven to be quite difficult to analyze.

\subsection{The action $S_{3}$}

First let us define the canonical momenta. Since the action (2.77) is independent of the time derivatives of $N, N^{i}, \zeta_{1}, \xi_{1}$ and $\xi_{2}$, their canonically conjugated momenta, $p_{N}, p_{i}$, $p_{\zeta_{1}}, p_{\xi_{1}}$ and $p_{\xi_{2}}$, respectively, are the primary constraints:

$$
p_{N} \approx 0, \quad p_{i}(\boldsymbol{x}) \approx 0, \quad p_{\zeta_{1}}(\boldsymbol{x}) \approx 0, \quad p_{\xi_{1}}(\boldsymbol{x}) \approx 0, \quad p_{\xi_{2}}(\boldsymbol{x}) \approx 0 .
$$

The momenta canonically conjugate to $g_{i j}$ and $\zeta_{2}$ are defined by

$$
\begin{aligned}
& p^{i j}=\frac{\delta S_{3}}{\delta \dot{g}_{i j}}=\sqrt{g}\left(\frac{1}{2 \kappa^{2}} \mathcal{G}^{i j k l} K_{k l}-\alpha U_{0}\left(\xi_{1} g^{i j} \nabla_{n} \zeta_{2}+\xi_{2} \mathcal{G}^{i j k l} K_{k l}\right)\right), \\
& p_{\zeta_{2}}=\frac{\delta S_{3}}{\delta \dot{\zeta}_{2}}=-\sqrt{g} 2 \alpha U_{0} \xi_{1} K .
\end{aligned}
$$

The Poisson bracket (PB) between the fields and the momenta are postulated in the conventional form. The nonvanishing $\mathrm{PBs}$ are defined (equal time $t$ is understood):

$$
\begin{array}{ll}
\left\{g_{i j}(\boldsymbol{x}), p^{k l}(\boldsymbol{y})\right\}=\delta_{(i}^{(k} \delta_{j}^{l)} \delta^{3}(\boldsymbol{x}-\boldsymbol{y}), & \left\{N, p_{N}\right\}=1, \\
\left\{N^{i}(\boldsymbol{x}), p_{j}(\boldsymbol{y})\right\}=\delta_{j}^{i} \delta^{3}(\boldsymbol{x}-\boldsymbol{y}), & \left\{\zeta_{1}(\boldsymbol{x}), p_{\zeta_{1}}(\boldsymbol{y})\right\}=\delta^{3}(\boldsymbol{x}-\boldsymbol{y}),
\end{array}
$$


and similarly for the fields $\xi_{1}, \zeta_{2}$ and $\xi_{2}$ as for $\zeta_{1}$. Recall that the lapse variable was constrained to be projectable, $N=N(t)$, due to the constraints (2.61) and $\phi=\phi(t)$.

In order to obtain the Hamiltonian, we perform the Legendre transform in the usual manner. First we solve $\dot{\zeta}_{2}$ and $\dot{g}_{i j}$ in terms of the canonical variables (the fields and their conjugated momenta):

$$
\begin{aligned}
\dot{\zeta}_{2} & =N^{i} \partial_{i} \zeta_{2}-\frac{N}{\sqrt{g} 3 \alpha U_{0} \xi_{1}}\left(p-\frac{1-2 \kappa^{2} \alpha U_{0} \xi_{2}}{2 \kappa^{2} \alpha U_{0} \xi_{1}} p_{\zeta_{2}}\right), \\
\dot{g}_{i j} & =2 N K_{i j}+2 D_{(i} N_{j)}, \\
K_{i j} & =\frac{1}{\sqrt{g}} \frac{2 \kappa^{2}}{1-2 \kappa^{2} \alpha U_{0} \xi_{2}} \mathcal{G}_{i j k l}\left[p^{k l}-\frac{g^{k l}}{3}\left(p-\frac{1-2 \kappa^{2} \alpha U_{0} \xi_{2}}{2 \kappa^{2} \alpha U_{0} \xi_{1}} p_{\zeta_{2}}\right)\right],
\end{aligned}
$$

where we denote $p=g_{i j} p^{i j}$. Then we define the total Hamiltonian by

$$
H_{3}=\int d^{3} \boldsymbol{x}\left(\dot{g}_{i j} p^{i j}+\dot{\zeta}_{2} p_{\zeta_{2}}+\lambda_{N} p_{N}+\lambda^{i} p_{i}+\lambda_{\zeta_{1}} p_{\zeta_{1}}+\lambda_{\xi_{1}} p_{\xi_{1}}+\lambda_{\xi_{2}} p_{\xi_{2}}\right)-L_{3}
$$

where $\lambda_{N}, \lambda^{i}, \lambda_{\zeta_{1}}, \lambda_{\xi_{1}}$ and $\lambda_{\xi_{2}}$ are Lagrange multiplier fields. We obtain the Hamiltonian by substituting (3.5)-(3.7) into (3.8):

$$
H_{3}=\int d^{3} \boldsymbol{x}\left(N \mathcal{H}_{0}^{3}+N^{i} \mathcal{H}_{i}^{3}+\lambda_{N} p_{N}+\lambda^{i} p_{i}+\lambda_{\zeta_{1}} p_{\zeta_{1}}+\lambda_{\xi_{1}} p_{\xi_{1}}+\lambda_{\xi_{2}} p_{\xi_{2}}\right)
$$

where we have defined

$$
\begin{aligned}
\mathcal{H}_{0}^{3} & =\frac{1}{\sqrt{g}} \frac{2 \kappa^{2}}{1-2 \kappa^{2} \alpha U_{0} \xi_{2}}\left[p_{i j} p^{i j}-\frac{1}{3} p^{2}-\frac{1}{3}\left(\frac{1-2 \kappa^{2} \alpha U_{0} \xi_{2}}{2 \kappa^{2} \alpha U_{0} \xi_{1}}\right) p p_{\zeta_{2}}+\frac{1}{6}\left(\frac{1-2 \kappa^{2} \alpha U_{0} \xi_{2}}{2 \kappa^{2} \alpha U_{0} \xi_{1}}\right)^{2} p_{\zeta_{2}}^{2}\right] \\
& +\sqrt{g}\left\{-\frac{1+2 \kappa^{2} \alpha U_{0} \xi_{2}}{2 \kappa^{2}} R+\alpha\left[\zeta_{1} \zeta_{2}+\xi_{1}\left(\zeta_{1}-2 U_{0} D^{i} D_{i} \zeta_{2}\right)+\xi_{2} \zeta_{2}\right]\right\} \\
\mathcal{H}_{i}^{3} & =-2 g_{i j} D_{k} p^{j k}+D_{i} \zeta_{2} p_{\zeta_{2}} \\
& =-2 g_{i j} \partial_{k} p^{j k}-\left(2 \partial_{j} g_{i k}-\partial_{i} g_{j k}\right) p^{j k}+\partial_{i} \zeta_{2} p_{\zeta_{2}} .
\end{aligned}
$$

Here we denote $p_{i j}=g_{i k} g_{j l} p^{k l} . \mathcal{H}_{i}^{3}$ are the momentum constraints - three constraints at every point on $\Sigma_{t}$. Because of the condition $N=N(t)$ the Hamiltonian constraint is the integral of $\mathcal{H}_{0}^{3}$ - a single global constraint.

The primary constraints (3.1) must be preserved under time evolution generated by 
the total Hamiltonian $H_{3}$ of the system:

$$
\begin{aligned}
\dot{p}_{N} & =\left\{p_{N}, H_{3}\right\}=-\int d^{3} \boldsymbol{x} \mathcal{H}_{0}^{3} \\
\dot{p}_{i} & =\left\{p_{i}, H_{3}\right\}=-\mathcal{H}_{i}^{3}, \\
\dot{p}_{\zeta_{1}} & =\left\{p_{\zeta_{1}}, H_{3}\right\}=-N\left\{\int d^{3} \boldsymbol{x} \mathcal{H}_{0}^{3}, p_{\zeta_{1}}\right\}=-N \sqrt{g} \alpha\left(\zeta_{2}+\xi_{1}\right) \\
\dot{p}_{\xi_{1}} & =\left\{p_{\xi_{1}}, H_{3}\right\}=-N\left\{\int d^{3} \boldsymbol{x} \mathcal{H}_{0}^{3}, p_{\xi_{1}}\right\} \\
& =-N\left[\frac{1}{\sqrt{g}} \frac{1}{3 \alpha U_{0} \xi_{1}^{2}}\left(p p_{\zeta_{2}}-\frac{1-2 \kappa^{2} \alpha U_{0} \xi_{2}}{2 \kappa^{2} \alpha U_{0} \xi_{1}} p_{\zeta_{2}}^{2}\right)+\sqrt{g} \alpha\left(\zeta_{1}-2 U_{0} D^{i} D_{i} \zeta_{2}\right)\right] \\
\dot{p}_{\xi_{2}} & =\left\{p_{\xi_{2}}, H_{3}\right\}=-N\left\{\int d^{3} \boldsymbol{x} \mathcal{H}_{0}^{3}, p_{\xi_{2}}\right\} \\
& =-N\left\{\frac{1}{\sqrt{g}}\left[\frac{4 \kappa^{4} \alpha U_{0}}{\left(1-2 \kappa^{2} \alpha U_{0} \xi_{2}\right)^{2}}\left(p_{i j} p^{i j}-\frac{1}{3} p^{2}\right)-\frac{p_{\zeta_{2}}^{2}}{6 \alpha U_{0} \xi_{1}^{2}}\right]+\sqrt{g} \alpha\left(\zeta_{2}-U_{0} R\right)\right\} .
\end{aligned}
$$

Therefore we impose the following secondary constraints:

$$
\begin{aligned}
\Phi_{0}^{3} & =\int d^{3} \boldsymbol{x} \mathcal{H}_{0}^{3} \approx 0, \quad \Phi_{i}^{3}(\boldsymbol{x})=\mathcal{H}_{i}^{3} \approx 0, \quad \Phi_{4}^{3}(\boldsymbol{x})=\zeta_{2}+\xi_{1} \approx 0 \\
\Phi_{5}^{3}(\boldsymbol{x}) & =\frac{1}{g}\left(p p_{\zeta_{2}}-\frac{1-2 \kappa^{2} \alpha U_{0} \xi_{2}}{2 \kappa^{2} \alpha U_{0} \xi_{1}} p_{\zeta_{2}}^{2}\right)+3 \alpha^{2} U_{0} \xi_{1}^{2}\left(\zeta_{1}-2 U_{0} D^{i} D_{i} \zeta_{2}\right) \approx 0 \\
\Phi_{6}^{3}(\boldsymbol{x}) & =\frac{1}{g}\left(p_{i j} p^{i j}-\frac{1}{3} p^{2}-\frac{1}{6}\left(\frac{1-2 \kappa^{2} \alpha U_{0} \xi_{2}}{2 \kappa^{2} \alpha U_{0} \xi_{1}}\right)^{2} p_{\zeta_{2}}^{2}\right)+\frac{\left(1-2 \kappa^{2} \alpha U_{0} \xi_{2}\right)^{2}}{4 \kappa^{4} U_{0}}\left(\zeta_{2}-U_{0} R\right) \approx 0 .
\end{aligned}
$$

The superscript 3 in constraints $\mathcal{H}_{0}^{3}, \mathcal{H}_{i}^{3}$ and $\Phi_{n}^{3}, n=0,1, \ldots, 6$ refers to the value of the critical exponent $z=3$ in the chosen model. Note that in the right-hand side of expressions like (3.18) we often omit the argument $\boldsymbol{x}$ when there is no risk of confusion. Evidently we are dealing with a more complicated structure of constraints than in GR or in (projectable) HL gravity. We will see that the constraints $\Phi_{n}^{3}(\boldsymbol{x}), n=4,5,6$ are second-class and they enable one to define the auxiliary fields $\xi_{1}, \zeta_{1}, \xi_{2}$ in terms of the dynamical fields $g_{i j}, \zeta_{2}$ and their canonically conjugated momenta $p^{i j}, p_{\zeta_{2}}$.

It is convenient to introduce a global smeared version of the momentum constraints (3.11):

$$
\Phi_{S}^{3}\left(\chi^{i}\right)=\int d^{3} \boldsymbol{x} \chi^{i} \mathcal{H}_{i}^{3}
$$

where $\chi^{i}(i=1,2,3)$ are arbitrary functions on $\Sigma_{t}$ which vanish rapidly enough at infinity. As the name suggests, the momentum constraints generate the infinitesimal spatial diffeomorphisms of the dynamical variables:

$$
\begin{aligned}
\left\{\Phi_{S}^{3}\left(\chi^{k}\right), g_{i j}\right\} & =-\chi^{k} \partial_{k} g_{i j}-g_{i k} \partial_{j} \chi^{k}-g_{j k} \partial_{i} \chi^{k}, \\
\left\{\Phi_{S}^{3}\left(\chi^{k}\right), p^{i j}\right\} & =-\partial_{k} \chi^{k} p^{i j}-\chi^{k} \partial_{k} p^{i j}+p^{i k} \partial_{k} \chi^{j}+p^{j k} \partial_{k} \chi^{i}, \\
\left\{\Phi_{S}^{3}\left(\chi^{i}\right), \zeta_{2}\right\} & =-\chi^{i} \partial_{i} \zeta_{2}, \\
\left\{\Phi_{S}^{3}\left(\chi^{i}\right), p_{\zeta_{2}}\right\} & =-\partial_{i} \chi^{i} p_{\zeta_{2}}-\chi^{i} \partial_{i} p_{\zeta_{2}} .
\end{aligned}
$$


Its PBs with the nondynamical fields and their canonically conjugated momenta vanish. However, when $\xi_{1}, \zeta_{1}, \xi_{2}$ are solved in terms of the dynamical variables, they behave as scalar fields, i.e. like $\zeta_{2}$ in Eq. (3.21). The momenta are tensor or scalar densities of weight -1 under spatial diffeomorphism, while the fields are regular tensors or scalars.

In order to check that the secondary constraints $\Phi_{I}^{3}(I=0,1,2, \ldots, 6)$ are preserved under time evolution, we need to evaluate their PBs with every constraint. First consider the momentum constraint (3.20). Its PB with the primary constraints (3.1) vanish. Since $\mathcal{H}_{0}^{3}$ is a scalar density of weight -1 under spatial diffeomorphism, its integral vanishes 4

$$
\left\{\Phi_{S}^{3}\left(\chi^{i}\right), \Phi_{0}^{3}\right\}=0
$$

The PB of the momentum constraint with itself forms the Lie algebra

$$
\left\{\Phi_{S}^{3}\left(\chi^{i}\right), \Phi_{S}^{3}\left(\psi^{i}\right)\right\}=\Phi_{S}^{3}\left(\chi^{j} \partial_{j} \psi^{i}-\psi^{j} \partial_{j} \chi^{i}\right) \approx 0
$$

Then consider the PBs with the rest of the secondary constraints in Eqs (3.17)-(3.19). We see that the constraints $\Phi_{n}^{3}(\boldsymbol{x}), n=4,5,6$ have been defined to be scalars under spatial diffeomorphism.

The Hamiltonian constraint $\Phi_{0}^{3}$ has vanishing PBs with $p_{N}$ and $p_{i}$. The PBs with the rest of the primary constraints were calculated in Eqs. (3.14)-(3.16), and they vanish due to the secondary constraints (3.17)-(3.19). Since the Hamiltonian constraint is global, its PB commutes with itself

$$
\left\{\Phi_{0}^{3}, \Phi_{0}^{3}\right\}=0 \text {. }
$$

Next consider the PBs with the secondary constraints $\Phi_{n}^{3}(\boldsymbol{x}), n=4,5,6$ :

$$
\left\{\Phi_{4}^{3}(\boldsymbol{x}), \Phi_{0}^{3}\right\}=-\frac{1}{\sqrt{g}} \frac{1}{3 \alpha U_{0} \xi_{1}}\left(p-\frac{1-2 \kappa^{2} \alpha U_{0} \xi_{2}}{2 \kappa^{2} \alpha U_{0} \xi_{1}} p_{\zeta_{2}}\right)
$$

and the PBs of the last two secondary constraints turn out to be rather complicated expressions:

$$
\begin{aligned}
&\left\{\Phi_{5}^{3}(\boldsymbol{x}), \Phi_{0}^{3}\right\}= \frac{1}{g^{3 / 2}} \frac{3 \kappa^{2}}{1-2 \kappa^{2} \alpha U_{0} \xi_{2}}\left[p_{i j} p^{i j}-\frac{1}{3} p^{2}+\frac{1}{3}\left(\frac{1-2 \kappa^{2} \alpha U_{0} \xi_{2}}{2 \kappa^{2} \alpha U_{0} \xi_{1}}\right) p p_{\zeta_{2}}\right. \\
&\left.-\frac{1}{2}\left(\frac{1-2 \kappa^{2} \alpha U_{0} \xi_{2}}{2 \kappa^{2} \alpha U_{0} \xi_{1}}\right)^{2} p_{\zeta_{2}}^{2}\right] p_{\zeta_{2}}+\frac{1}{\sqrt{g}}\left\{\frac{1+2 \kappa^{2} \alpha U_{0} \xi_{2}}{4 \kappa^{2}} R\right. \\
&-\alpha\left[\frac{3}{2} \zeta_{1} \zeta_{2}+\right.\left.\xi_{1}\left(\frac{3}{2} \zeta_{1}-2 U_{0} D^{i} D_{i} \zeta_{2}\right)+\frac{3}{2} \xi_{2} \zeta_{2}+U_{0}\left(D_{i} \xi_{1} D^{i} \zeta_{2}-2 D^{i} D_{i} \xi_{2}\right)\right] p_{\zeta_{2}} \\
&\left.+\alpha\left(p-2 \frac{1-2 \kappa^{2} \alpha U_{0} \xi_{2}}{2 \kappa^{2} \alpha U_{0} \xi_{1}} p_{\zeta_{2}}\right)\left(2 U_{0} D^{i} D_{i} \xi_{1}-\zeta_{1}-\xi_{2}\right)\right\} \\
&+\frac{1}{\sqrt{g}} 6 \alpha^{2} U_{0}^{2} \xi_{1}^{2}\left[\left(D^{i} D^{j} \zeta_{2}-D^{(i} \zeta_{2} D^{j)}+\frac{1}{2} g^{i j} D^{k} D_{k}\right)\right. \\
& \frac{2 \kappa^{2}}{1-2 \kappa^{2} \alpha U_{0} \xi_{2}}\left(2 p_{i j}-\frac{g_{i j}}{3}\left(2 p+\frac{1-2 \kappa^{2} \alpha U_{0} \xi_{2}}{2 \kappa^{2} \alpha U_{0} \xi_{1}} p_{\zeta_{2}}\right)\right) \\
&\left.+D^{i} D_{i} \frac{1}{3 \alpha U_{0} \xi_{1}}\left(p-\frac{1-2 \kappa^{2} \alpha U_{0} \xi_{2}}{2 \kappa^{2} \alpha U_{0} \xi_{1}} p_{\zeta_{2}}\right)\right]
\end{aligned}
$$

\footnotetext{
${ }^{4}$ For a scalar density $\psi_{w}$ of weight $w$ we find: $\left\{\Phi_{S}^{3}\left(\chi^{i}\right), \int d^{3} \boldsymbol{x} \psi_{w}\right\}=-(w+1) \int d^{3} \boldsymbol{x} \chi^{i} \partial_{i} \psi_{w}$.
} 
and

$$
\begin{aligned}
&\left\{\Phi_{6}^{3}(\boldsymbol{x}), \Phi_{0}^{3}\right\}=\frac{1}{g^{3 / 2}} \frac{p_{\zeta_{2}}}{\alpha U_{0} \xi_{1}}\left[p_{i j} p^{i j}-\frac{1}{3} p^{2}-\frac{1}{6}\left(\frac{1-2 \kappa^{2} \alpha U_{0} \xi_{2}}{2 \kappa^{2} \alpha U_{0} \xi_{1}}\right)^{2} p_{\zeta_{2}}\right] \\
&+\frac{1}{\sqrt{g}}\left\{\frac{1+2 \kappa^{2} \alpha U_{0} \xi_{2}}{2 \kappa^{2}}\left(\frac{2}{3} R p-R_{i j} p^{i j}\right)-\alpha \xi_{2} \zeta_{2} p\right. \\
&+\alpha U_{0}\left[2\left(p^{i j}-\frac{1}{3} p g^{i j}\right)\left(D_{i} D_{j} \xi_{2}-2 D_{(i} \xi_{1} D_{j} \zeta_{2}\right)-2 \xi_{1}\left(4 p^{i j}-\frac{1}{3} p g^{i j}\right) D_{i} D_{j} \zeta_{2}\right] \\
&+\frac{\alpha}{3}\left(\zeta_{1}+\xi_{2}-2 U_{0} D^{i} D_{i} \xi_{1}\right)\left(\frac{1-2 \kappa^{2} \alpha U_{0} \xi_{2}}{2 \kappa^{2} \alpha U_{0} \xi_{1}}\right)^{2} p_{\zeta_{2}} \\
&- \frac{\left(1-2 \kappa^{2} \alpha U_{0} \xi_{2}\right)^{2}}{4 \kappa^{4}}\left[\frac{1}{3 \alpha U_{0} \xi_{1}}\left(p-\frac{1-2 \kappa^{2} \alpha U_{0} \xi_{2}}{2 \kappa^{2} \alpha U_{0} \xi_{1}} p_{\zeta_{2}}\right)\right. \\
&-U_{0}\left(R_{i j}-D_{i} D_{j}\right.\left.\left.\left.+g_{i j} D^{k} D_{k}\right) \frac{2 \kappa^{2}}{1-2 \kappa^{2} \alpha U_{0} \xi_{2}}\left(2 p_{i j}-\frac{g_{i j}}{3}\left(2 p+\frac{1-2 \kappa^{2} \alpha U_{0} \xi_{2}}{2 \kappa^{2} \alpha U_{0} \xi_{1}} p_{\zeta_{2}}\right)\right)\right]\right\}
\end{aligned}
$$

The nonvanishing PBs between $\Phi_{n}^{3}(\boldsymbol{x}), n=4,5,6$ and the primary constraints (3.1) are

$$
\begin{aligned}
& \left\{\Phi_{4}^{3}(\boldsymbol{x}), p_{\xi_{1}}(\boldsymbol{y})\right\}=\delta^{3}(\boldsymbol{x}-\boldsymbol{y}), \quad\left\{\Phi_{5}^{3}(\boldsymbol{x}), p_{\zeta_{1}}(\boldsymbol{y})\right\}=3 \alpha^{2} U_{0} \xi_{1}^{2} \delta^{3}(\boldsymbol{x}-\boldsymbol{y}), \\
& \left\{\Phi_{5}^{3}(\boldsymbol{x}), p_{\xi_{1}}(\boldsymbol{y})\right\}=\left(\frac{1}{g} \frac{1-2 \kappa^{2} \alpha U_{0} \xi_{2}}{2 \kappa^{2} \alpha U_{0} \xi_{1}} p_{\zeta_{2}}+6 \alpha^{2} U_{0} \xi_{1} \zeta_{1}\right) \delta^{3}(\boldsymbol{x}-\boldsymbol{y}), \\
& \left\{\Phi_{5}^{3}(\boldsymbol{x}), p_{\xi_{2}}(\boldsymbol{y})\right\}=\frac{1}{g} \frac{1}{\xi_{1}} p_{\zeta_{2}}^{2} \delta^{3}(\boldsymbol{x}-\boldsymbol{y}), \\
& \left\{\Phi_{6}^{3}(\boldsymbol{x}), p_{\xi_{1}}(\boldsymbol{y})\right\}=\frac{1}{g} \frac{1}{3 \xi_{1}}\left(\frac{1-2 \kappa^{2} \alpha U_{0} \xi_{2}}{2 \kappa^{2} \alpha U_{0} \xi_{1}}\right)^{2} p_{\zeta_{2}}^{2} \delta^{3}(\boldsymbol{x}-\boldsymbol{y}), \\
& \left\{\Phi_{6}^{3}(\boldsymbol{x}), p_{\xi_{2}}(\boldsymbol{y})\right\}=\left(\frac{1}{g} \frac{1}{3 \xi_{1}} \frac{1-2 \kappa^{2} \alpha U_{0} \xi_{2}}{2 \kappa^{2} \alpha U_{0} \xi_{1}} p_{\zeta_{2}}^{2}-\frac{\alpha}{\kappa^{2}}\left(1-2 \kappa^{2} \alpha U_{0} \xi_{2}\right)\left(\zeta_{2}-U_{0} R\right)\right) \delta^{3}(\boldsymbol{x}-\boldsymbol{y}) .
\end{aligned}
$$

Now we can consider the stability of the secondary constraints. First we obtain that the Hamiltonian constraint and the momentum constraints are preserved under time evolution:

$$
\begin{aligned}
& \dot{\Phi}_{0}^{3}=\left\{\Phi_{0}^{3}, H_{3}\right\}=\int d^{3} \boldsymbol{x} \sqrt{g}\left(\lambda_{\zeta_{1}} \alpha \Phi_{4}^{3}+\lambda_{\xi_{1}} \frac{1}{3 \alpha U_{0} \xi_{1}^{2}} \Phi_{5}^{3}+\lambda_{\xi_{2}} \frac{4 \kappa^{4} \alpha U_{0}}{\left(1-2 \kappa^{2} \alpha U_{0} \xi_{2}\right)^{2}} \Phi_{6}^{3}\right) \approx 0, \\
& \dot{\Phi}_{S}^{3}\left(\chi^{i}\right)=\left\{\Phi_{S}^{3}\left(\chi^{i}\right), H_{3}\right\}=\left\{\Phi_{S}^{3}\left(\chi^{i}\right), \Phi_{S}^{3}\left(N^{i}\right)\right\}=\Phi_{S}^{3}\left(\chi^{j} \partial_{j} N^{i}-N^{j} \partial_{j} \chi^{i}\right) \approx 0 .
\end{aligned}
$$

The rest of the secondary constraints evolve under time as follows:

$$
\begin{aligned}
\dot{\Phi}_{4}^{3}(\boldsymbol{x}) & =\left\{\Phi_{4}^{3}(\boldsymbol{x}), H_{3}\right\}=-\frac{1}{\sqrt{g}} \frac{N}{3 \alpha U_{0} \xi_{1}}\left(p-\frac{1-2 \kappa^{2} \alpha U_{0} \xi_{2}}{2 \kappa^{2} \alpha U_{0} \xi_{1}} p_{\zeta_{2}}\right)+N^{i} \partial_{i} \Phi_{4}^{3}+\lambda_{\xi_{1}}, \\
\dot{\Phi}_{5}^{3}(\boldsymbol{x}) & =\left\{\Phi_{5}^{3}(\boldsymbol{x}), H_{3}\right\}=N\left\{\Phi_{5}^{3}(\boldsymbol{x}), \Phi_{0}^{3}\right\}+N^{i} \partial_{i} \Phi_{5}^{3}+\lambda_{\zeta_{1}} 3 \alpha^{2} U_{0} \xi_{1}^{2} \\
& +\lambda_{\xi_{1}}\left(\frac{1}{g} \frac{1-2 \kappa^{2} \alpha U_{0} \xi_{2}}{2 \kappa^{2} \alpha U_{0} \xi_{1}} p_{\zeta_{2}}+6 \alpha^{2} U_{0} \xi_{1} \zeta_{1}\right)+\lambda_{\xi_{2}} \frac{1}{g} \frac{1}{\xi_{1}} p_{\zeta_{2}}^{2}, \\
\dot{\Phi}_{6}^{3}(\boldsymbol{x}) & =\left\{\Phi_{6}^{3}(\boldsymbol{x}), H_{3}\right\}=N\left\{\Phi_{6}^{3}(\boldsymbol{x}), \Phi_{0}^{3}\right\}+N^{i} \partial_{i} \Phi_{6}^{3}+\lambda_{\xi_{1}} \frac{1}{g} \frac{1}{3 \xi_{1}}\left(\frac{1-2 \kappa^{2} \alpha U_{0} \xi_{2}}{2 \kappa^{2} \alpha U_{0} \xi_{1}}\right)^{2} p_{\zeta_{2}}^{2} \\
& +\lambda_{\xi_{2}}\left(\frac{1}{g} \frac{1}{3 \xi_{1}} \frac{1-2 \kappa^{2} \alpha U_{0} \xi_{2}}{2 \kappa^{2} \alpha U_{0} \xi_{1}} p_{\zeta_{2}}^{2}-\frac{\alpha}{\kappa^{2}}\left(1-2 \kappa^{2} \alpha U_{0} \xi_{2}\right)\left(\zeta_{2}-U_{0} R\right)\right),
\end{aligned}
$$


where Eqs. (3.26) and (3.27) are understood. We see that imposing $\dot{\Phi}_{n}^{3}(\boldsymbol{x}) \approx 0, n=4,5,6$ in Eq. (3.30) fixes the Lagrange multipliers $\lambda_{\xi_{1}}, \lambda_{\zeta_{1}}$ and $\lambda_{\xi_{2}}$. Thus no more constraints are required to fulfill the consistency conditions for the secondary constraints. Hence we have obtained a stable constraint surface in the phase space.

As noted above, we may use the constraints $\Phi_{n}^{3}(\boldsymbol{x}), n=4,5,6$ to eliminate the auxiliary fields $\zeta_{1}, \xi_{1}$ and $\xi_{2}$. To this end, we can set the second-class constraints $p_{\zeta_{1}}, p_{\xi_{1}}, p_{\xi_{2}}$ and $\Phi_{n}^{3}(\boldsymbol{x}), n=4,5,6$ to vanish strongly by introducing the Dirac bracket, and then solve the auxiliary fields in terms of the dynamical variables from Eqs. (3.17)-(3.19). This eliminates the fields $\zeta_{1}, \xi_{1}, \xi_{2}$ and their canonically conjugated momenta from the phase space of the system.

Let us then count the number of physical degrees of freedom (physical d.o.f.) by using Dirac's formula:

$$
\begin{array}{r}
\#(\text { physical d.o.f. })=\frac{1}{2}[\#(\text { canonical variables })-2 \times \#(\text { first-class constraints }) \\
-\#(\text { second-class constraints })] .
\end{array}
$$

For nonpropagating zero modes we have 28 canonical variables $\left(N, N^{i}, g_{i j}, \xi_{1}, \zeta_{1}, \xi_{2}, \zeta_{2}\right.$, and their conjugated momenta), 8 first-class constraints $\left(p_{N}, p_{i}, \Phi_{0}^{3}, \mathcal{H}_{i}^{3}\right)$, and 6 secondclass constraints $\left(p_{\zeta_{1}}, p_{\xi_{1}}, p_{\xi_{2}}, \Phi_{4}^{3}, \Phi_{5}^{3}, \Phi_{6}^{3}\right)$. This yields

$$
\#(\text { nonpropagating physical d.o.f. })=\frac{1}{2}(28-16-6)=3
$$

for the ( $\boldsymbol{x}$-independent) zero modes. For propagating modes we have 26 canonical variables $\left(N^{i}, g_{i j}, \xi_{1}, \zeta_{1}, \xi_{2}, \zeta_{2}\right.$, and their conjugated momenta), 6 first-class constraints $\left(p_{i}, \mathcal{H}_{i}^{3}\right)$, and 6 second-class constraints $\left(p_{\zeta_{1}}, p_{\xi_{1}}, p_{\xi_{2}}, \Phi_{4}^{3}, \Phi_{5}^{3}, \Phi_{6}^{3}\right)$. Thus for propagating modes we obtain

$$
\#(\text { propagating physical d.o.f. })=\frac{1}{2}(26-12-6)=4 \text {. }
$$

For comparison, there are only two physical degrees of freedom in GR. As another comparison, our analysis shows that CRG with the condition $\phi=\phi(t)$ has one more physical degree of freedom than projectable HL gravity, which has 2 zero modes and 3 propagating modes. Interestingly, the number of physical modes is exactly the same as in the modified $F(R)$ HL gravity [1, 2, 11]. One extra physical degree of freedom has its origin in the higher order time derivatives present in the CRG action. The other extra propagating mode is caused by the projectability condition similarly as in HL gravity. Such extra degrees of freedom can be problematic since they may generate extra (long range) forces that are not in agreement with observations. One may be able to bring the number of physical degrees of freedom closer to that of GR by introducing some extra gauge symmetry, which generates some new constraints. Another possible way to deal with the extra propagating mode is to make the scalar field $\zeta_{2}$ massive.

\subsection{The action $S_{4}$}

Since the action (2.78) is independent of the time derivatives of $N, N^{i}, \eta$ and $\xi$, their canonically conjugated momenta, $p_{N}, p_{i}, p_{\eta}$ and $p_{\xi}$ respectively, are the primary constraints:

$$
p_{N} \approx 0, \quad p_{i}(\boldsymbol{x}) \approx 0, \quad p_{\eta}(\boldsymbol{x}) \approx 0, \quad p_{\xi}(\boldsymbol{x}) \approx 0
$$


The momenta conjugate to $g_{i j}$ and $\zeta$ are defined by

$$
\begin{aligned}
p^{i j} & =\frac{\delta S_{4}}{\delta \dot{g}_{i j}}=\sqrt{g}\left(\frac{1}{2 \kappa^{2}} \mathcal{G}^{i j k l} K_{k l}+\alpha U_{0}\left(2 \eta g^{i j} \nabla_{n} \zeta+\xi \mathcal{G}^{i j k l} K_{k l}\right)\right), \\
p_{\zeta} & =\frac{\delta S_{4}}{\delta \dot{\zeta}}=\sqrt{g} 4 \alpha U_{0} \eta K .
\end{aligned}
$$

The PB is postulated similarly as in Eq. (3.4).

Solving $\dot{\zeta}$ and $\dot{g}_{i j}$ in terms of the canonical variables gives

$$
\begin{aligned}
\dot{\zeta} & =N^{i} \partial_{i} \zeta+\frac{N}{\sqrt{g} 6 \alpha U_{0} \eta}\left(p+\frac{1+2 \kappa^{2} \alpha U_{0} \xi}{4 \kappa^{2} \alpha U_{0} \eta} p_{\zeta}\right) \\
\dot{g}_{i j} & =2 N K_{i j}+2 D_{(i} N_{j)}, \\
K_{i j} & =\frac{1}{\sqrt{g}} \frac{1+2 \kappa^{2} \alpha U_{0} \xi}{2 \kappa^{2}} \mathcal{G}_{i j k l}\left[p^{k l}+\frac{g^{k l}}{3}\left(p+\frac{1+2 \kappa^{2} \alpha U_{0} \xi}{4 \kappa^{2} \alpha U_{0} \eta} p_{\zeta}\right)\right]
\end{aligned}
$$

The total Hamiltonian is obtained similarly as before:

$$
H_{4}=\int d^{3} \boldsymbol{x}\left(N \mathcal{H}_{0}^{4}+N^{i} \mathcal{H}_{i}^{4}+\lambda_{N} p_{N}+\lambda^{i} p_{i}+\lambda_{\eta} p_{\eta}+\lambda_{\xi} p_{\xi}\right)
$$

where the Hamiltonian and momentum constraints are defined by

$$
\begin{aligned}
\mathcal{H}_{0}^{4} & =\frac{1}{\sqrt{g}} \frac{2 \kappa^{2}}{1+2 \kappa^{2} \alpha U_{0} \xi}\left[p_{i j} p^{i j}-\frac{1}{3} p^{2}+\frac{1}{3}\left(\frac{1+2 \kappa^{2} \alpha U_{0} \xi}{4 \kappa^{2} \alpha U_{0} \eta}\right) p p_{\zeta}+\frac{1}{6}\left(\frac{1+2 \kappa^{2} \alpha U_{0} \xi}{4 \kappa^{2} \alpha U_{0} \eta}\right)^{2} p_{\zeta}^{2}\right] \\
& -\sqrt{g}\left[\frac{1-2 \kappa^{2} \alpha U_{0} \xi}{2 \kappa^{2}} R+\alpha\left(\eta^{2}-4 U_{0} \eta D^{i} D_{i} \zeta+\xi \zeta\right)\right] \\
\mathcal{H}_{i}^{4} & =-2 g_{i j} D_{k} p^{j k}+D_{i} \zeta p_{\zeta} \\
& =-2 g_{i j} \partial_{k} p^{j k}-\left(2 \partial_{j} g_{i k}-\partial_{i} g_{j k}\right) p^{j k}+\partial_{i} \zeta p_{\zeta}
\end{aligned}
$$

The primary constraints (3.34) must be preserved under the time evolution generated by the total Hamiltonian $H_{4}$ of the system:

$$
\begin{aligned}
\dot{p}_{N} & =\left\{p_{N}, H_{4}\right\}=-\int d^{3} \boldsymbol{x} \mathcal{H}_{0}^{4} \\
\dot{p}_{i} & =\left\{p_{i}, H_{4}\right\}=-\mathcal{H}_{i}^{4} \\
\dot{p}_{\eta} & =\left\{p_{\eta}, H_{4}\right\}=-N\left\{\int d^{3} \boldsymbol{x} \mathcal{H}_{0}^{4}, p_{\eta}\right\} \\
& =N\left[\frac{1}{\sqrt{g}} \frac{1}{6 \alpha U_{0} \eta^{2}}\left(p p_{\zeta}+\frac{1+2 \kappa^{2} \alpha U_{0} \xi}{4 \kappa^{2} \alpha U_{0} \eta} p_{\zeta}^{2}\right)+\sqrt{g} 2 \alpha\left(\eta-2 U_{0} D^{i} D_{i} \zeta\right)\right], \\
\dot{p}_{\xi} & =\left\{p_{\xi}, H_{4}\right\}=-N\left\{\int d^{3} \boldsymbol{x} \mathcal{H}_{0}^{4}, p_{\xi}\right\} \\
& =N\left\{\frac{1}{\sqrt{g}}\left[\frac{4 \kappa^{4} \alpha U_{0}}{\left(1+2 \kappa^{2} \alpha U_{0} \xi\right)^{2}}\left(p_{i j} p^{i j}-\frac{1}{3} p^{2}\right)-\frac{p_{\zeta}^{2}}{24 \alpha U_{0} \eta^{2}}\right]+\sqrt{g} \alpha\left(\zeta-U_{0} R\right)\right\} .
\end{aligned}
$$


Therefore we impose the following secondary constraints:

$$
\begin{aligned}
\Phi_{0}^{4} & =\int d^{3} \boldsymbol{x} \mathcal{H}_{0}^{4} \approx 0, \quad \Phi_{i}^{4}(\boldsymbol{x})=\mathcal{H}_{i}^{4} \approx 0 \\
\Phi_{4}^{4}(\boldsymbol{x}) & =\frac{1}{g}\left(p p_{\zeta}+\frac{1+2 \kappa^{2} \alpha U_{0} \xi}{4 \kappa^{2} \alpha U_{0} \eta} p_{\zeta}^{2}\right)+12 \alpha^{2} U_{0} \eta^{2}\left(\eta-2 U_{0} D^{i} D_{i} \zeta\right) \approx 0 \\
\Phi_{5}^{4}(\boldsymbol{x}) & =\frac{1}{g}\left(p_{i j} p^{i j}-\frac{1}{3} p^{2}-\frac{1}{6}\left(\frac{1+2 \kappa^{2} \alpha U_{0} \xi}{4 \kappa^{2} \alpha U_{0} \eta}\right)^{2} p_{\zeta}^{2}\right)+\frac{\left(1+2 \kappa^{2} \alpha U_{0} \xi\right)^{2}}{4 \kappa^{4} U_{0}}\left(\zeta-U_{0} R\right) \approx 0 .
\end{aligned}
$$

The constraint structure is quite similar to the case $z=3$ we discussed above. The only major difference is that there is one auxiliary field less in the case $z=4$ than in the case $z=3$, and therefore there are two second-class constraints less than in the case $z=3$ - one primary constraint and one secondary constraint. The consistency conditions $\dot{\Phi}_{n}^{4}(\boldsymbol{x}) \approx 0, n=4,5$ can be satisfied by fixing the Lagrange multipliers $\lambda_{\eta}$ and $\lambda_{\xi}$. Thus we again obtain a stable constraint surface. Once imposed, the second-class constraints (3.48) and (3.49) define the auxiliary fields $\eta$ and $\xi$ in terms of the dynamical variables. The number of physical degrees of freedom is the same as in the case $z=3$.

\section{Conclusion and discussion}

Let us summarize our approach and results. Following the proposal of Ref. [4], we considered that the exotic perfect fluid of CRG is generated by a scalar field $\phi$ that is constrained by Eq. (2.5). We obtained the ADM representations of such CRG actions for the supposedly power-counting renormalizable $z=3$ and super-renormalizable $z=4$ cases. The corresponding Lagrangians were found to contain time derivatives of the dynamical fields up to fourth order. In order to obtain Lagrangians with kinetic parts quadratic in the first order time derivatives, we introduced some additional scalar fields and took advantage of the constraint (2.5) by choosing $\partial^{\mu} \phi$ to be parallel to the normal of the spatial hypersurfaces. The Hamiltonian formalism for the space-time decomposed actions was developed. We showed that both actions have a consistent set of constraints. All but one of the additional scalar fields turn out to be auxiliary, which can be eliminated by imposing the second-class constraints of the systems. The number of physical degrees of freedom was found to be 3 for zero modes and 4 for propagating modes. Thus we can conclude that the advantage of retaining general covariance and local Lorentz invariance in the formulation of CRG comes with the price of an extra physical degree of freedom - compared to HL gravity. This is a consequence of the higher order nature of the CRG action. Compared to GR we found two extra propagating physical degrees of freedom. One may be able to reduce the number of physical degrees of freedom closer to that of GR by introducing some extra gauge symmetry or by making some of the fields massive. These points, however, were not pursued further in this paper.

It would be interesting to use the Pons formalism [20] for the Hamiltonian analysis of CGR in order to gain deeper understanding of these higher order derivative theories, and with the hope that a more conclusive statement on their nature could be drawn. It would enable one to analyze CRG without imposing the projectability condition on the scalar field $\phi$, and consequently on the lapse $N$. Another possible prospect is the application of perturbative constraints to CRG. This could enable one to eliminate the possible problems caused by the higher order time derivatives. It would also be interesting to see whether 
the "covariant vector gravity" proposed in Ref. [5] could provide even more fruitful results compared to the present scalar formulation, since unlike $\partial_{\mu} \phi$, a vector field $A_{\mu}$ does not involve time derivatives.

Acknowledgements We wish to thank Sergei Odintsov for correspondence and invaluable comments. The support of the Academy of Finland under the Projects No. 136539 and No. 140886 is gratefully acknowledged. M. O. is supported by the Jenny and Antti Wihuri Foundation and by the Finnish Cultural Foundation.

\section{References}

[1] M. Chaichian, S. Nojiri, S. D. Odintsov, M. Oksanen and A. Tureanu, "Modified $F(R)$ Hořava-Lifshitz gravity: a way to accelerating FRW cosmology", Class. Quant. Grav. 27, 185021 (2010), arXiv:1001.4102 [hep-th].

[2] S. Carloni. M. Chaichian, S. Nojiri, S. D. Odintsov, M. Oksanen and A. Tureanu, "Modified first-order Hořava-Lifshitz gravity: Hamiltonian analysis of the general theory and accelerating FRW cosmology in power-law $F(R)$ model", Phys. Rev. D 82, 065020 (2010), arXiv:1003.3925 [hep-th].

[3] S. Nojiri and S. D. Odintsov, "Covariant renormalizable gravity and its FRW cosmology", Phys. Rev. D 81, 043001 (2010), arXiv:0905.4213 [hep-th].

[4] S. Nojiri and S. D. Odintsov, "A proposal for covariant renormalizable field theory of gravity", Phys. Lett. B 691, 60 (2010), arXiv:1004.3613 [hep-th].

[5] S. Nojiri and S. D. Odintsov, "Covariant power-counting renormalizable gravity: Lorentz symmetry breaking and accelerating early-time FRW universe", Phys. Rev. D 83023001 (2011), arXiv:1007.4856 [hep-th].

[6] S. Nojiri and S. D. Odintsov, "Unified cosmic history in modified gravity: from $F(R)$ theory to Lorentz non-invariant models", Phys. Rep. (doi:10.1016/j.physrep.2011.04. 001), arXiv:1011.0544 [gr-qc].

[7] P. Hořava, "Quantum Gravity at a Lifshitz Point", Phys. Rev. D 79, 084008 (2009), arXiv:0901.3775 [hep-th].

[8] T. P. Sotiriou, M. Visser and S. Weinfurtner, "Phenomenologically Viable Lorentz-Violating Quantum Gravity", Phys. Rev. Lett. 102, 251601 (2009), arXiv:0904.4464 [hep-th],

[9] D. Blas, O. Pujolàs and S. Sibiryakov, "A healthy extension of Hořava gravity", Phys. Rev. Lett. 104, 181302 (2010), arXiv:0909.3525 [hep-th].

[10] M. Henneaux, A. Kleinschmidt and G. L. Gómez, "A dynamical inconsistency of Hořava gravity", Phys. Rev. D 81, 064002 (2010), arXiv:0912.0399 [hep-th],

[11] M. Chaichian, M. Oksanen and A. Tureanu, "Hamiltonian analysis of nonprojectable modified $F(R)$ Hořava-Lifshitz gravity", Phys. Lett. B 693, 404 (2010), arXiv:1006.3235 [hep-th]. 
[12] R. L. Arnowitt, S. Deser and C. W. Misner, "The dynamics of general relativity", arXiv:gr-qc/0405109, originally in Gravitation: An Introduction to Current Research, L. Witten ed., John Wiley \& Sons Inc., New York, London, 1962. Republished in Gen. Relativ. Gravit. 40, 1997 (2008).

[13] R. M. Wald, General Relativity, University of Chicago Press, Chicago and London, 1984.

[14] É. Gourgoulhon, "3+1 Formalism and Bases of Numerical Relativity", arXiv:gr-qc/0703035.

[15] M. Ostrogradski, Mem. Ac. St. Petersbourg VI 4, 385 (1850).

[16] D. A. Eliezer and R. P. Woodard, "The problem of nonlocality in string theory", Nucl. Phys. B 325, 389 (1989).

[17] P. A. M. Dirac, "Generalized Hamiltonian dynamics", Can. J. Math. 2, 129 (1950).

[18] P. A. M. Dirac, Proc. R. Soc. London Sr. A 246, 326 (1958).

[19] P. A. M. Dirac, Lectures on Quantum Mechanics, Yeshiva University, New York, 1964.

[20] J. M. Pons, "Ostrogradski's Theorem for Higher-Order Singular Lagrangians", Lett. Math. Phys. 17, 181 (1989).

[21] J. Llosa and J. Vives, "Hamiltonian formalism for nonlocal Lagrangians", J. Math. Phys. 35, 2856 (1994).

[22] J. Z. Simon, "Higher derivative Lagrangians, nonlocality, problems and solutions", Phys. Rev. D 41, 3720 (1990).

[23] S. Capozziello, J. Matsumoto, S. Nojiri and S. D. Odintsov, "Dark energy from modified gravity with Lagrange multipliers", Phys. Lett. B 693, 198 (2010), arXiv:1004.3691 [hep-th],

[24] J. Klusoň, "Hamiltonian Analysis of Lagrange Multiplier Modified Gravity", arXiv:1009.6067 [hep-th].

[25] R. P. Woodard, "Avoiding Dark Energy with 1/R Modifications of Gravity", Lect. Notes Phys. 720, 403 (2007), arXiv:astro-ph/0601672. 